\title{
Impact of co-morbidities on the mortality of patients with status epilepticus and the utility of RCBI score in evaluating the mortality of status epilepticus
}

\section{Yu Zhang}

Sichuan University West China Hospital

\section{Deng Chen}

Sichuan University West China Hospital

\section{Li-na Zhu}

Sichuan University West China Hospital

ling liu ( $\nabla$ zjllxx1968@163.com )

Sichuan University West China Hospital Department of Neurology https://orcid.org/0000-0003-09276372

Research article

Keywords: status epilepticus, complications, prognosis, RCBI

Posted Date: January 10th, 2020

DOl: https://doi.org/10.21203/rs.2.20623/v1

License: (c) (i) This work is licensed under a Creative Commons Attribution 4.0 International License. Read Full License 


\section{Abstract}

Background: To investigate the influential factors of complications on prognosis of patients with status epilepticus, modify the Complication Burden Index $\triangle \mathrm{CBI} \otimes$ into the Rankin CBIهRCBI区, and analyze its practicability in status epilepticus in western China.

Method: A total of 396 patients with status epilepticus were studied from December 2016 to January 2019 in West China Hospital. The clinical data were collected, including demographic characteristics, status epilepticus characteristics. Statistical analysis was performed using SPSS 22.0 and MedCalc ROC『and logistic regression was used to analyze the influencing factors of hospitalization death and poor prognosis (GOS scale is 1-3).

Results: Of the 396 patients with status epilepticus included in the study, 43 (10.9\%) died in hospital and $114(28.8 \%)$ had poor prognosis. Using ROC curve analysis, when RCBI > 3, the area under the ROC curve of hospitalization death was $0.914, p<0.0001$; When RCBI $>3$, the area under ROC curve for poor prognosis was $0.882, p<0.0001$. There were 327 people with convulsive status epilepticus, including 41 deaths. When $\mathrm{RCBI}>3$, the area under the hospital mortality curve was $0.915(p<0.0001)$. A total of 100 patients had a poor prognosis. When $\mathrm{RCBI}>3$, the area under the poor prognosis curve was 0.867 $(p<0.0001)$.

Conclusions: The hospital mortality rate of patients with status epilepticus is $10.9 \%$. RCBI $>3$ points had a certain significance for predicting hospitalization death and poor prognosis of status epilepticus. There were no significant differences in RCBI scales for convulsive status epilepticus and non-convulsive status epilepticus.

\section{Background}

Status epilepticus is one of the most common critical illnesses in neurology. Its main features are persistent seizures, which are characterized by unpredictable, complex conditions and rapid progression. Patients with status epilepticus have high disability and mortality. [3]. According to foreign literature reports, the mortality rate of epileptic seizures is $3-33 \%$, and the mortality rate of convulsive epilepsy in southwest China is $5.4-15.8 \%[1,8]$. Therefore, it is especially important to quickly assess the patient's condition and predict the mortality rate. It is possible to objectively formulate and revise the medical care plan, improve the quality of medical care, and make rational use of medical resources. In recent years, there have been many studies on the prognosis of status epilepticus, including a variety of scales and biological markers $[3-5,8,11]$. At present, five commonly used international scoring scales for predicting the prognosis of patients with status epilepticus include the epidemiology-based mortality scale in status

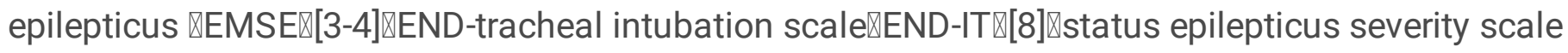

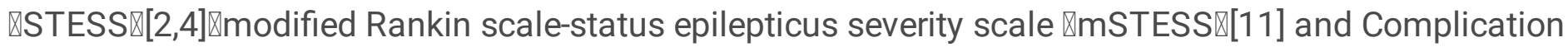
Burden Index $\mathbb{C B} \mathbb{C}[5]$. Systemic complications are very common in patients with status epilepticus, and the types and number of complications may influence the prognosis of patients [5,9-10,16-19,24]. In 2018, 
Leena et al [5] proposed the $\mathrm{CBI}$ and evaluated patients for 13 types of complications: respiratory system, cardiovascular system, nervous system, kidney, liver, coagulation function, gastrointestinal and musculoskeletal system, and electrolyte/acid-base balance, infection, hypoglycemia/hyperglycemia, skin/allergic reaction, and mental disorders. Using the receiver operating characteristic (ROC) curve, the maximum $\mathrm{CBI}$ scale was 13 points, the average $\mathrm{CBI}$ was 3.8 points, and the cutoff point for poor prediction function was $₫ 3$ points[5]. The $\mathrm{CBI}$ scale established by Leena et al. included a small number of patients, and only included convulsive epileptic patients with seizure time $>30$ min.

Therefore, the purpose of this study was to investigate the influencing factors of co-morbidity of patients with epileptic status on their prognosis, modify $\mathrm{CBI}$ scale into the RCBI scale, and evaluate whether it is applicable to the population in western China, as well as its feasibility in non-convulsive epileptic status.

\section{Methods}

1.1. Subjects: This study included 396 patients from the West China Hospital of Sichuan University from December 2016 to January 2019, from patients with emergency, NICU, neurology, and neurosurgical epilepsy. All patients met the latest diagnostic criteria for status epilepticus (2015) of the International League Against Epilepsy (ILAE). The clinical data collected included demographic characteristics, status epilepticus characteristics such as duration of epilepsy, and all comorbidities. The International League Against Epilepsy (ILAE) is the latest diagnostic criteria for status epilepticus (2015). According to the standards of the Helsinki Declaration, this study strictly abides by the principle of voluntary and informed patients. If patients did not have autonomy, researchers obtained the voluntary and informed consent of their immediate family members.

- Inclusion criteria: 1. Conform to the clinical diagnostic criteria for epilepsy published by the International Anti-epilepsy Alliance [1]; 2. Patients with status epilepticus who voluntarily provide their informed consent; 3 . Age $\geq 18$ years old; 4 . Patients who have received EEG examination; 5 . All patients were treated according to the continuous epilepsy treatment process of the American Antiepilepsy Association in 2016 [7].

- Exclusion criteria: 1. EEG is the epileptic state of the interictal EEG; 2. Patients with status epilepticus without informed consent; 3 . Patients who do not have complete data.

1.2 Research methods: The study included 33 comorbidities recommended by St. Germaine-Smith [25] and 13 complications included in the $\mathrm{CBI}$ scale that were summarized and screened at discharge, such as hyperglycemia and hypoglycemia, which can be counted as electrolytes. Electrolyte imbalance and acidbase imbalance can be counted as one category, and the etiology of epileptic seizures needs to be removed. Therefore, comorbidity is defined as a disease that is comorbid during the status epilepticus but does not include the factors that cause status epilepticus, including skin allergy, musculoskeletal-related diseases, digestive system diseases, thyroid function diseases, respiratory system diseases, immune system diseases, kidney and urinary system diseases, electrolyte/acid-base balance disorders, hypoglycemia/hyperglycemia, hypoproteinemia, infection, blood system diseases, mental diseases, 
nervous system diseases (excluding the causes of status epilepticus, such as brain tumors, acute stroke, etc.), and cardiovascular system diseases. Regardless of the severity of the common disease or the involvement of multiple systems, it is only calculated once, and the total number of diseases is up to 15 (Table 1). The outcome variables were hospitalization death and poor prognosis. The evaluation index of poor prognosis was GOS scale of 1-3.

1.3 Using SPSS 22.0 to analyze data, P value $<0.05$ was statistically significant; using MedCalc statistical analysis software, the area under the ROC curve [6] reflected the diagnostic value of the scale: $(0.50$, $0.70)$, indicating that the diagnostic value is lower; $(0.70,0.90)$, indicating a moderate diagnostic value; 0.90 or more indicating a higher diagnostic value, and $\mathrm{P}<0.05$ was statistically significant. Sensitivity (true positive rate) is plotted as the ordinate on the ROC curve, while 1-specificity (false positive rate) is plotted as the abscissa. Maximizing the true positive rate while minimizing the false positive rate gives the best diagnostic criteria for the scale.

\section{Result}

1. General information of the 396 patients with status epilepticus was included in the study. Forty-one patients $(10.3 \%)$ died in hospital, and $114(28.8 \%)$ had poor prognosis. Among them, $219(55.3 \%)$ were male patients and 177 were female, with an age range of 18-96 years. Among 396 patients with status epilepticus, 327 (82.6\%) had convulsive status epilepticus, and 69 (17.4\%) had non-convulsive status epilepticus. Two hundred fifty-one (63.4\%) had no history of epilepsy, 170 (42.9\%) had EEG abnormalities (defined as focal epilepsy or epileptic discharge, diffuse or multifocal slow wave rhythm), 105 (26.5\%) had head MRI abnormalities, and 98 (24.7\%) had tracheal intubation (Table 2).

2. Analysis of risk factors for death in hospital and poor prognosis in patients with status epilepticus. Among 396 patients with status epilepticus included in the study, 4 had skin allergy, 7 had musculoskeletal-related diseases, 48 had digestive system diseases, 17 had thyroid function diseases, 73 had respiratory system diseases, 10 had immune system diseases, 52 had kidney and urinary system diseases, 53 had electrolyte/acid-base balance disorders, 39 had hypoglycemia/hyperglycemia, 46 had hypoproteinemia, 187 had infection, 25 had coagulation and blood diseases, 14 had mental diseases, 62 had nervous system diseases, 73 had cardiovascular system diseases, and a total of 41 had died. The binary logistic regression analysis showed that digestive system $(P=0.000)$, respiratory system $(P=0.001)$, immune system $(P=0.021)$, kidney and urinary system $(P=0.000)$, and electrolyte/acid-base imbalance $(P=0.001)$ and infection $(P=0.015)$ were the risk factors for hospitalization death in the epileptic state. Musculoskeletal diseases $(P=0.003)$, digestive system $(P=0.000)$, thyroid function $(P=0.017)$, respiratory system $(P=0.000)$, immune system $(P=0.000)$, kidney/urinary system $(P=0.000)$, electrolyte/acid-base imbalance $(P=0.000)$, hypoglycemia/hyperglycemia $(P=0.012)$, infection $(P=0.000)$, blood system $(P=0.005)$, nervous system $(P=0.000)$, and cardiovascular system diseases $(P=0.000)$ are the risk factors for poor prognosis of patients with status epilepticus (Table 3 ) 
3. Comorbidity analysis of patients with status epilepticus. Among 396 patients, 21 patients suffered from 0 comorbidity, of which 0 died in hospital and 0 had poor prognosis. There were 38 patients with one kind of comorbidity, 2 died in hospital, and 8 had poor prognosis. There were 71 cases of both comorbidities, 3 died in hospital, and 7 had poor prognosis. There were 124 patients with 3 kinds of comorbidities, 21 died in hospital, and 55 had poor prognosis. There were 98 patients with 4 kinds of comorbidities, 7 died in hospital, and 25 had poor prognosis. There were 29 patients with 5 kinds of comorbidities, of which 6 died in hospital and 12 had poor prognosis. There were 10 patients with 6 kinds of comorbidities $\$ of which 2 died in hospital and 4 had poor prognosis. There were 4 patients with 7 kinds of comorbidities, including 1 patient died in hospital and 2 patients had poor prognosis. There were 2 patients suffering from 9 kinds of comorbidities, including 1 death in hospital and 1 case of poor prognosis (Table 2). The average number of common diseases is 2.98. A total of 43 people died. MedCalc ROC curve was used for analysis. When RCBI $>3$, the specificity was $71.37 \%$, the sensitivity was $97.67 \%$, the AUC was 0.914 , the standard error was 0.020 , and the P value was $<0.0001,95 \%$ confidence interval was $0.881-0.939$, and the accuracy was high (Figure 1-1). A total of 114 patients had a poor prognosis. When $\mathrm{RCBI}>3$, the specificity was $81.56 \%$, the sensitivity was $79.82 \%$, the AUC was 0.882 , the standard error was 0.016 , the $P$ value was $<0.0001$, the $95 \%$ confidence interval was $0.846-0.912$ \and the accuracy was medium (Figure 1-2 ).

4. Comparative analysis of persistent status of convulsive epilepsy and non-convulsive epilepsy. There were 327 people with convulsive status epilepticus, including 41 deaths. When $\mathrm{RCB}>3$, the specificity was $70.38 \%$, the sensitivity is $97.50 \%$, the AUC was 0.915 , the standard error was 0.021 , the P value is $<0.0001$, the $95 \%$ confidence interval was $0.880-0.943$, and the accuracy was high (Figure $2-1$ ). The prognosis was poor in 100 patients. When $\mathrm{RCBI}>3$, the specificity was $79.74 \%$, the sensitivity was $78.00 \%$, the AUC was 0.867 , the standard error was 0.018 , the $P$ value was $<0.0001$, the $95 \%$ confidence interval was $0.825-0.902$. The accuracy was medium (Figure 2-2). There were 43 nonconvulsive epilepsy states, including 2 deaths. When RCBI>3, the specificity was $75.76 \%$, the sensitivity is $100.00 \%$, the AUC was 0.919 , the standard error was 0.057 , and the P value was $<0.0001$. The $95 \%$ confidence interval was $0.828-0.971$, and the accuracy was high (Figure $3-1$ ). The prognosis included 14 patients. When RCBI>3, the specificity was $89.09 \%$, the sensitivity was $92.86 \%$, and the AUC was 0.955 . The standard error was 0.024 , the $P$ value is $<0.0001$, the $95 \%$ confidence interval was 0.876-0.990, and the accuracy was high (Figure 3-2).

\section{Discussion}

Status epilepticus is one of the most common neurological critical illnesses, often leading to permanent neurological damage, with high morbidity and mortality $[1,12-15,20-23]$. The mortality rate of convulsive epilepsy in southwestern China is 15.4-15.8\% [8]. Of the 396 patients enrolled in the study, 43 were hospitalized, and the mortality rate was approximately $10.9 \%$, which was consistent with the mortality rate of previous studies in Southwest China. 
This study showed that the digestive system, respiratory system, immune system, kidney and urinary system, electrolyte/acid-base imbalance, and infection are the risk factors for hospitalization death in status epilepticus. The musculoskeletal system diseases, digestive system, thyroid function, respiratory system, immune system, kidney/urinary system, electrolyte/acid-base imbalance, hypoglycemia/hyperglycemia, infection, blood system, nervous system, and cardiovascular system diseases are risk factors for poor prognosis of patients with status epilepticus. Therefore, we improved the $\mathrm{CBI}$ scale and added three new complications: immune system diseases, thyroid function diseases and hypoproteinemia, because these three diseases also have certain influence on the prognosis of patients, such as hyperthyroidism/hypofunction, etc. In addition, the liver system and gastrointestinal diseases are combined into the digestive system to avoid double counting. Coagulation function should be expanded to include diseases of the blood system,such as anemia. The renal system and urinary system are combined into one item. This study did not consider tumor (excluding the cause of status epilepticus), which obviously affects the prognosis of patients, because it probably existed before status epilepticus.

The average total number of epileptic patients included in this study is 2.98 , which is somewhat different from the average $\mathrm{CBI}$ of 3.8 obtained by Leena et al. The reasons may be as follows: 1 . The patients with status epilepticus included in this study are included in the study according to the latest definition of status epilepticus [1], and the patients with status epilepticus lasting for 5-30 min, that is T1 time, are included in the study. The sample size and scope of patients included in the study are larger than those of previous studies, and the number of patients suffering from epilepsy in T1 time is less, the hospitalization time is shorter, and the prognosis is better. 2. Patients with non-convulsive status epilepticus were included in this study. 3 . With the continuous development of medical technology, the awareness of medical personnel in the prevention and treatment of complications has continuously improved, and complications have generally decreased. 4. In this study, all factors that may be the cause of the status epilepticus, such as tumor, metabolic brain, autoimmune encephalitis and acute stroke, were excluded. 5. When complications involve multiple systems, the standard of only one calculation shall be strictly followed. For example, the calculation shall only be carried out once when patients suffer from urinary tract infection, lung infection, and blood system infection at the same time. This study shows that the cutoff point of RCBI scale for predicting hospital death and poor prognosis of patients with status epilepsy is 3 , which is consistent with the results obtained by Leena et al.

For convulsive status epilepticus, the cut-off point for predicting hospital death and poor prognosis is 3 , and for non-convulsive status epilepticus, the cut-off point for predicting hospital death and poor prognosis is also 3. In this study, patients with non-convulsive status epilepticus were analyzed separately, which proves that it has certain predictive value for hospital death and poor prognosis of patients with non-convulsive status epilepticus, and there is no significant difference compared with convulsive status epilepticus. The RCBI scale is simple to operate and can be completed in a short time, compared with the previous EMSE and STESS scores, the effect of complications on the prognosis of patients with epilepsy status during hospitalization is more focused, which enriches the scale for 
predicting the prognosis of the status epilepticus and fills the gap in the non-convulsive status epilepticus prediction scale.

This was a single-center study. The patients included in the study were all from a hospital in western China, which may have certain selection bias. This study was scaled by a doctor's own judgment, and there may be some deviation. In the subsequent study, it is expected that two doctors will scale at the same time to obtain more accurate scoring results. Therefore, more scales or indicators may be needed to evaluate the prognosis of patients with epilepsy, and large-scale multicenter studies are needed to further verify our findings.

\section{Conclusion}

The in-hospital mortality rate of patients with status epilepticus in western China is approximately $10.9 \%$, and $\mathrm{RCBI}>3$, which is of certain significance for predicting in-hospital death and poor prognosis of status epilepticus. Regarding the status of convulsive epilepsy and non-convulsive epilepsy, RCBI has no significant difference in predicting hospital death and poor prognosis(GOS 1-3).

\section{Declarations}

\section{Ethics approval and consent to participate}

The protocol was approved by the West China hospital ethics Committee

\section{Consent for publication}

I can confirm I have consent for publication and study participants was written]

\section{Availability of data and material}

The datasets generated or analysed during the current study are not publicly available due not have consent from all patients, but are available from the corresponding author on reasonable request.

\section{Competing interests}

The authors declare no conflicts of interest.

\section{Funding}

This study was funded by the Science \& Technology Department of Sichuan Province [grant number 2018SZ0166] in the analysis.

\section{Authors' contributions}

YZ collects data and writes articles 
CD and ZLN are responsible for collation and statistics

LL reviews articles

All authors have read and approved the manuscript.

\section{Acknowledgements}

We would like to thank American Journal Experts for assistance with revising the grammar, syntax and phrasing of the manuscript.

\section{References}

1. Trinka E, Cock H, Hesdorffer D, Rossetti AO, Scheffer IE, Shinnar S, et al. A definition and classification of status epilepticus - report of the ILAE Task Force on Classification of Status Epilepticus. Epilepsia. 2015;56(10):1515-23

2. Rossetti AO, Logroscino G, Milligan TA, Michaelides C, Ruffieux C, Bromfield EB. Status epilepticus severity scale (STESS): a tool to orient early treatment strategy. J Neurol 2008;255(10):1561-6.

3. Leitinger M, Höller Y, Kalss G, Rohracher A, Novak HF, Höfler J. Epidemiology-based mortality scale in status epilepticus (EMSE). Neurocrit Care 2015;22:273-82.

4. Kang BS, Kim DW, Kim KK, Moon HJ, Kim Y-S, Kim HK, et al. Prediction of mortality and functional outcome from status epilepticus and independent external validation of STESS and EMSE scales. Crit Care 2015;20(1):25.

5. Kamppi L, Ritvanen J, Strbian D, Mustonen H, Soinila S. Complication Burden Index-A tool for comprehensive evaluation of the effect of complications on functional outcome after status epilepticus. Epilepsia. 2018;00:1-6.

6. Hanley JA, McNeil BJ. The meaning and use of the area under a receiver operating characteristic (ROC) curve. Radiology. 1982;143:29-36.

7. Glauser T, Shinnar S, Gloss D, et al. Evidence-Based Guideline: Treatment of Convulsive StatusEpilepticus in Children and Adults: Report of the Guideline Committee of the American Epilepsy Society[J]. Epilepsy Currents, 2016, 16(1): 48-61.

8. Gao Q, Ou-Yang T-P, Sun XI, et al. Prediction of functional outcome in patients with convulsive status epilepticus: the END-IT scale. Crit Care. 2016;20:46.

9. Sokic DV, Jankovic SM, Vojvodic NM, et al. Etiology of a short-term mortality in the group of 750 patients with 920 episodes of status epilepticus within a period of 10 years (19881997). Seizure. 2009;18:215-9.

10. Sutter R, Tschudin-Sutter S, Grize L, et al. Associations between infections and clinical outcome parameters in status epilepticus: a retrospective 5-year cohort study. Epilepsia. 2012;53:1489-97.

11. Gonzalez-Cuevas M, Santamarina E, Toledo M, et al. A new clinical scale for the prognosis of status epilepticus in adults. Eur J Neurol. 2016;23:1534-40. 
12. Legriel S, Mouvillier B, Bele N, et al. Outcomes in 140 critically ill patients with status epilepticus. Intensive Care Med. 2008;34:476-80.

13. Madžar D, Geyer A, Knappe RU, et al. Association of seizure duration and outcome in refractory status epilepticus. J Neurol. 2016;263:485-91.

14. Kowalski RG, Ziai WC, Rees RN, et al. Third-line antiepileptic therapy and outcome in status epilepticus: the impact of vasopressor use and prolonged mechanical ventilation. Crit Care Med. 2012;40:2677-84.

15. Hocker S, Britton JW, Mandrekar JN, et al. Predictors of outcome in refractory status epilepticus. JAMA Neurol. 2013;70:72-7.

16. Zelano J, Möller J, Dobesberger J, et al. Infections in status epilepticus: a retrospective 5-year cohort study. Seizure. 2014;23:603-6.

17. Hocker S, Prasad A, Rabinstein AA. Cardiac injury in refractory status epilepticus. Epilepsia. 2013;54:518-22.

18. Rosenow F, Hamer HM, Knake S. The epidemiology of convulsive and nonconvulsive status epilepticus. Epilepsia. 2007;48:82-4.

19. Belluzo M, Furlanis G, Stragapede L, et al. Role of comorbidities and in-hospital complications in short-term status epilepticus outcome. Clin Neurol Neurosurg. 2016;154:13-8.

20. Koubeissi M, Alshekhlee A. In-hospital mortality of generalized convulsive status epilepticus. Neurology. 2007;69:886-93.

21. Hocker S. Systemic complications of status epilepticus - An update. Epilepsy Behav. 2015;49:83-7.

22. Lai $\mathrm{A}$, Outin $\mathrm{H}$, Jabot $\mathrm{J}$, et al. Functional outcome of prolonged refractory status epilepticus. Crit Care. 2015;19:199. .

23. Sutter R, Kaplan PW, Rüegg S. Independent external validation of the status epilep- ticus severity scale. Crit Care Med 2013;41(12):e475-9

24. Alvarez V, Januel JM, Burnand B, Rossetti AO. Role of comorbidities in outcome prediction after status epilepticus. Epilepsia 2012;53(5):89-92.

25. St Germaine-Smith C , Liu M F , Quan H, et al. Development of an epilepsy-specific risk adjustment comorbidity index[J]. Epilepsia, 2011, 52(12):2161-2167.

\section{Tables}




\begin{tabular}{|ll|}
\hline comorbidity & Score \\
\hline skin allergy, & 1 \\
\hline musculoskeletal-related diseases & 1 \\
\hline digestive system diseases & 1 \\
\hline thyroid function diseases & 1 \\
\hline respiratory system diseases & 1 \\
\hline immune system diseases & 1 \\
\hline kidney and urinary system diseases & 1 \\
\hline electrolyte/acid-base balance disorders & 1 \\
\hline hypoglycemia/hyperglycemia & 1 \\
\hline hypoproteinemia & 1 \\
\hline infection & 1 \\
\hline coagulation and blood diseases & 1 \\
\hline mental diseases & 1 \\
\hline nervous system diseases & 1 \\
\hline cardiovascular system diseases & 1 \\
\hline Total score & 15 \\
\hline
\end{tabular}

Table1 $₫$ Categories in the Rankin Computation Burden Index $\triangle \mathrm{RCBI} \bigotimes$ 


\begin{tabular}{|c|c|c|c|}
\hline & Total $₫ 396 \rrbracket$ & $H D \otimes 43 / 10.9 \% \rrbracket$ & $\mathrm{PP} \otimes 114 / 28.8 \% \rrbracket$ \\
\hline Gender $\rrbracket$ male $₫ \bigotimes$ & $219 \rrbracket 55.30 \% \rrbracket$ & & \\
\hline Age $\rrbracket$ years $\bigotimes$ range $\rrbracket$ & $18-94$ & & \\
\hline Duration from SE to death & & Omin-15days & \\
\hline \multicolumn{4}{|l|}{ SE dynamics } \\
\hline CSE & $327 \llbracket 82.6 \% \rrbracket$ & $41 \rrbracket 10.4 \% \rrbracket$ & $100 \rrbracket 25.3 \% \rrbracket$ \\
\hline T1 time SE & $140 \rrbracket 35.4 \% \rrbracket$ & $7 \rrbracket 1.8 \% \bigotimes$ & $20 \rrbracket 5.1 \% \rrbracket$ \\
\hline T2 time SE & $106 \rrbracket 26.8 \% \rrbracket$ & $10 \rrbracket 2.5 \% \rrbracket$ & $36 \otimes 9.1 \% \rrbracket$ \\
\hline Refractory SE & $77 \rrbracket 19.4 \% \bigotimes$ & $21 \otimes 5.3 \% \rrbracket$ & $40 \otimes 10.1 \% \bigotimes$ \\
\hline SRSE & $4 \rrbracket 1 \% \rrbracket$ & $3 \rrbracket 0.8 \% \rrbracket$ & $1 \rrbracket 0.3 \% \rrbracket$ \\
\hline NCSE & $69 \rrbracket 17.4 \% \rrbracket$ & $2 \varangle 0.5 \% \rrbracket$ & $14 \llbracket 3.5 \% \rrbracket$ \\
\hline First episode of SE & $251 \rrbracket 63.4 \% \bigotimes$ & $41 \otimes 10.4 \% \rrbracket$ & $80 \rrbracket 20.2 \% \rrbracket$ \\
\hline EEG abnormal & $170 \rrbracket 42.9 \% \rrbracket$ & $31 \otimes 7.8 \% \bigotimes$ & $79 \otimes 19.9 \% \rrbracket$ \\
\hline MRI abnormal & $105 \rrbracket 26.5 \% \rrbracket$ & $15 \llbracket 3.8 \% \rrbracket$ & $67 \rrbracket 16.9 \% \rrbracket$ \\
\hline Need for tracheal intubation & $98 \rrbracket 24.7 \% \rrbracket$ & $40 \otimes 10.1 \% \rrbracket$ & $47 \rrbracket 11.9 \% \rrbracket$ \\
\hline \multicolumn{4}{|l|}{$\mathrm{RCBI}$} \\
\hline 0 & $21 \rrbracket 5.3 \% \rrbracket$ & 0 & 0 \\
\hline 1 & $38 \otimes 9.6 \% \square$ & $2 \bowtie 0.5 \% \rrbracket$ & $8 \bowtie 2.0 \% \rrbracket$ \\
\hline 2 & $71 \otimes 17.9 \% \bigotimes$ & $3 \bowtie 0.8 \% \rrbracket$ & $7 \rrbracket 1.8 \% \bigotimes$ \\
\hline 3 & $124 \llbracket 31.3 \% \rrbracket$ & $21 \rrbracket 5.3 \% \bigotimes$ & $55 \rrbracket 13.9 \% \bigotimes$ \\
\hline 4 & $98 \rrbracket 24.7 \% \rrbracket$ & $7 \rrbracket 1.8 \% \rrbracket$ & $25 \rrbracket 6.3 \% \rrbracket$ \\
\hline 5 & $29 \rrbracket 7.3 \% \rrbracket$ & $6 \bigotimes 1.5 \% \rrbracket$ & $12 \rrbracket 3.0 \% \rrbracket$ \\
\hline 6 & $10 \otimes 2.5 \% \rrbracket$ & $2 \varangle 0.5 \% \rrbracket$ & $4 \bigotimes 1 \% \bigotimes$ \\
\hline 7 & $4 \rrbracket 1 \% \rrbracket$ & 1ख0.3\%》 & $2 ه 0.5 \% \bigotimes$ \\
\hline 9 & $2 \varangle 0.5 \% \rrbracket$ & $1 \rrbracket 0.3 \% \rrbracket$ & $1 \rrbracket 0.3 \% \rrbracket$ \\
\hline
\end{tabular}

Table 2: Demographic and clinical data of patients included

HD :hospital deaths $₫ P P \llbracket$ poor prognosis $\ S E \rrbracket s t a t u s$ epilepticus 
SRSE\super refractory status epilepticus $\$

\begin{tabular}{|c|c|c|c|c|c|}
\hline comorbidity & Amount & $\begin{array}{l}\mathrm{HD} P- \\
\text { value }\end{array}$ & $\mathrm{HD} \mathrm{Cl}$ & $\begin{array}{l}\text { PP P- } \\
\text { value }\end{array}$ & $\mathrm{PP} \mathrm{Cl}$ \\
\hline skin allergy, & 4 & 0.957 & $\begin{array}{l}0.069- \\
37.701\end{array}$ & 0.171 & $\begin{array}{l}0.385- \\
220.745\end{array}$ \\
\hline musculoskeletal-related diseases & 7 & 0.999 & - & 0.003 & $\begin{array}{l}3.955- \\
666.084\end{array}$ \\
\hline digestive system diseases & 48 & 0.000 & $\begin{array}{l}3.054- \\
23.035\end{array}$ & 0.000 & $\begin{array}{l}5.981- \\
67.826\end{array}$ \\
\hline thyroid function diseases & 17 & 0.998 & - & 0.017 & $\begin{array}{l}1.503- \\
60.992\end{array}$ \\
\hline respiratory system diseases & 73 & 0.001 & $\begin{array}{l}1.968- \\
11.681\end{array}$ & 0.000 & $\begin{array}{l}9.922- \\
71.984\end{array}$ \\
\hline immune system diseases & 10 & 0.021 & $\begin{array}{l}1.374- \\
49.969\end{array}$ & 0.000 & $\begin{array}{l}8.123- \\
439.976\end{array}$ \\
\hline $\begin{array}{l}\text { kidney and urinary system } \\
\text { diseases }\end{array}$ & 52 & 0.000 & $\begin{array}{l}2.518- \\
20.801\end{array}$ & 0.000 & $\begin{array}{l}2.500- \\
24.648\end{array}$ \\
\hline $\begin{array}{l}\text { electrolyte/acid-base balance } \\
\text { disorders }\end{array}$ & 53 & 0.001 & $\begin{array}{l}2.173- \\
16.247\end{array}$ & 0.000 & $\begin{array}{l}5.115- \\
47.996\end{array}$ \\
\hline hypoglycemia/hyperglycemia & 39 & 0.265 & $\begin{array}{l}0.557- \\
8.367\end{array}$ & 0.012 & $\begin{array}{l}1.454- \\
19.966\end{array}$ \\
\hline hypoproteinemia & 46 & 0.335 & $\begin{array}{l}0.558- \\
5.548\end{array}$ & 0.997 & - \\
\hline infection & 187 & 0.015 & $\begin{array}{l}1.233- \\
7.126\end{array}$ & 0.000 & $\begin{array}{l}2.164- \\
12.202\end{array}$ \\
\hline coagulation and blood diseases & 25 & 0.942 & $\begin{array}{l}0.287- \\
3.829\end{array}$ & 0.005 & $\begin{array}{l}2.173- \\
68.859\end{array}$ \\
\hline mental diseases & 14 & 0.992 & $\begin{array}{l}0.104- \\
9.397\end{array}$ & 0.070 & $\begin{array}{l}0.880- \\
25.279\end{array}$ \\
\hline nervous system diseases & 62 & 0.511 & $\begin{array}{l}0.335- \\
3.004\end{array}$ & 0.000 & $\begin{array}{l}2.197- \\
12.306\end{array}$ \\
\hline cardiovascular system diseases & 73 & 0.996 & $\begin{array}{l}0.335- \\
1.723\end{array}$ & 0.000 & $\begin{array}{l}3.101- \\
21.129\end{array}$ \\
\hline
\end{tabular}

Table 3: Analysis of Risk Factors for hospital Death and Poor Prognosis in Status Epilepsy HD :hospital deaths $\ P P \unrhd$ poor prognos 
Figures

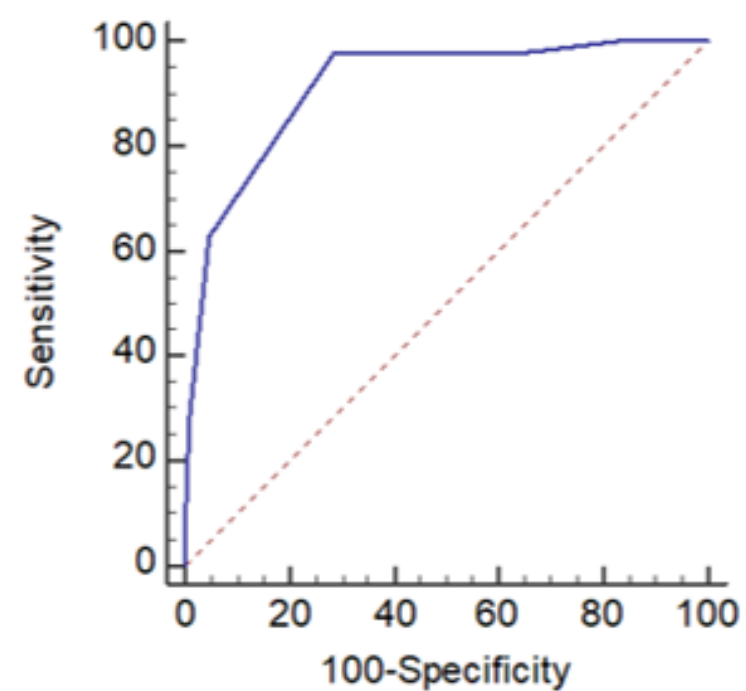

Figure1-1 Total hospital deaths

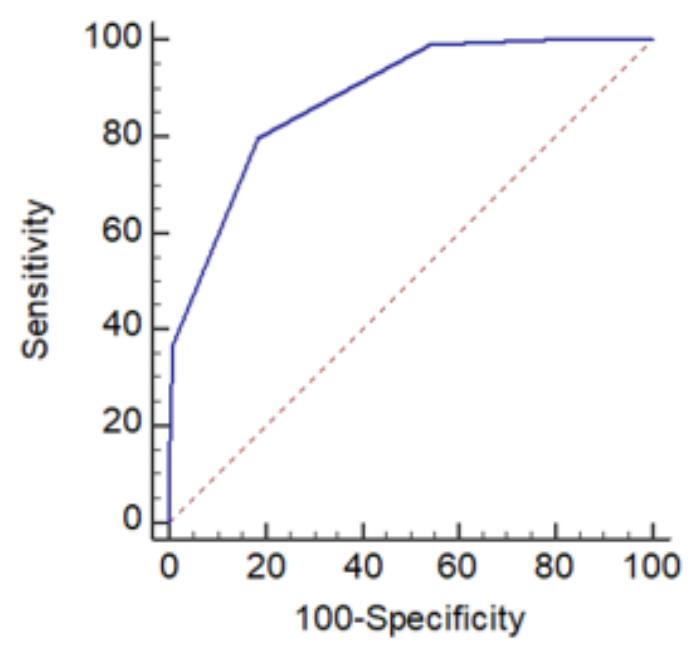

Figure1-2 Total poor prognosis

\section{Figure 1}

Total hospital deaths and poor prognosis

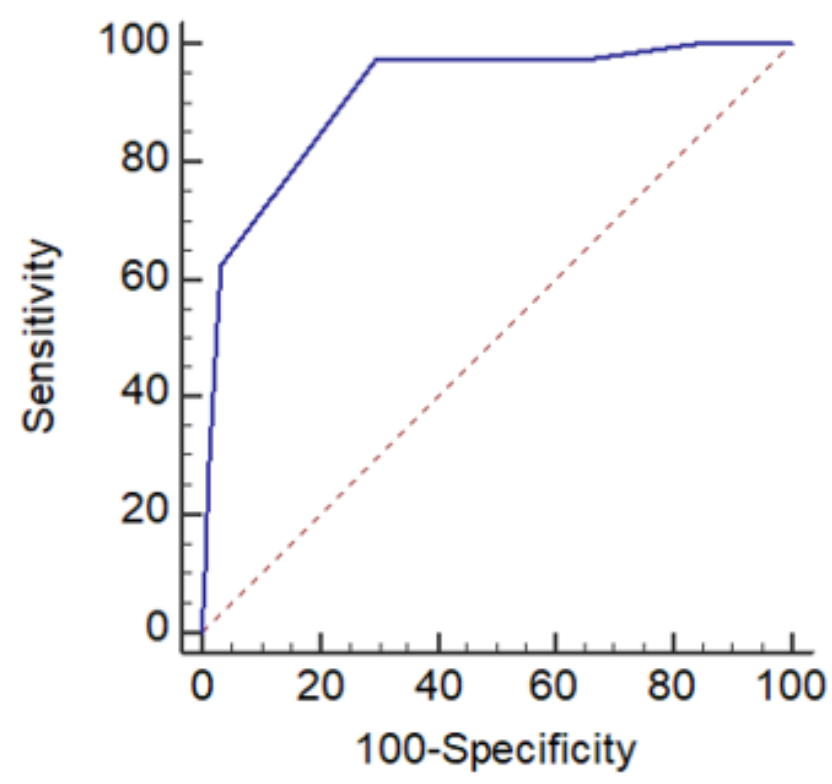

Figure2-1 CSE hospital deaths

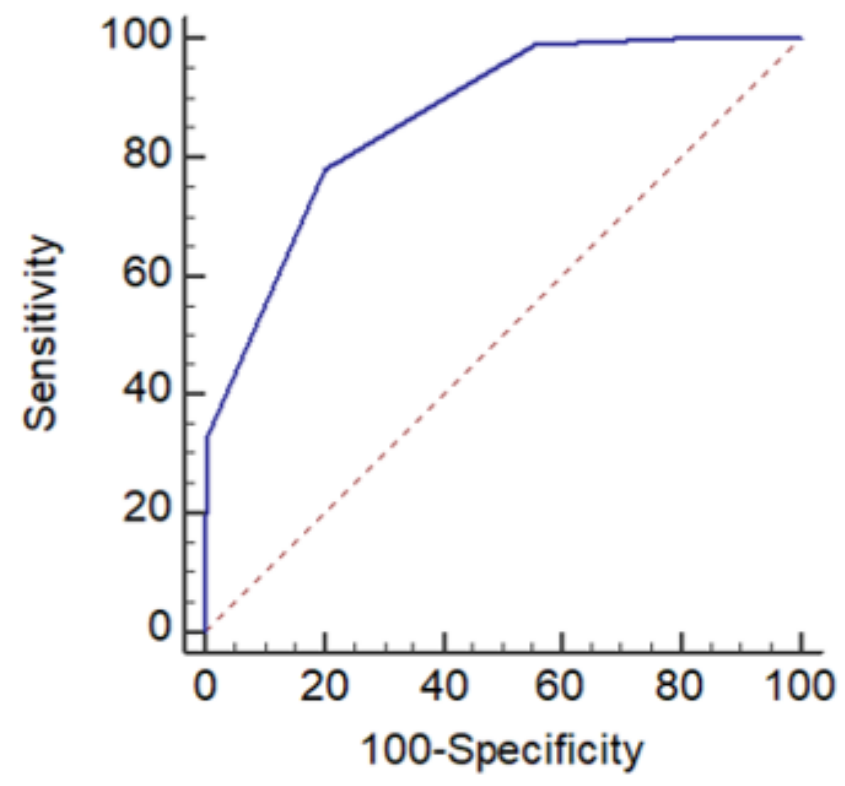

Figure2-2 CSE poor prognosis

Figure 2

CSE hospital deaths and poor prognosis 


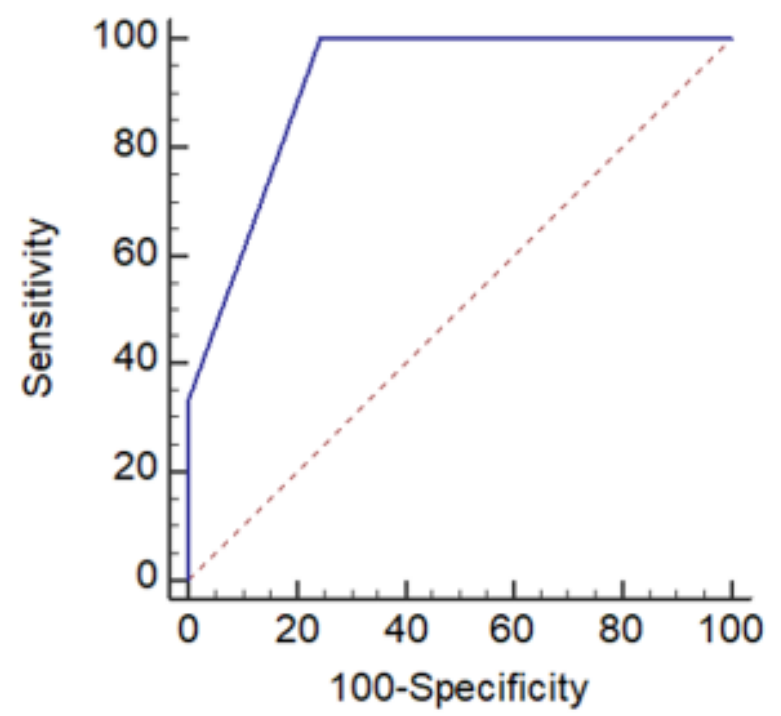

Figure3-1 NCSE hospital deaths

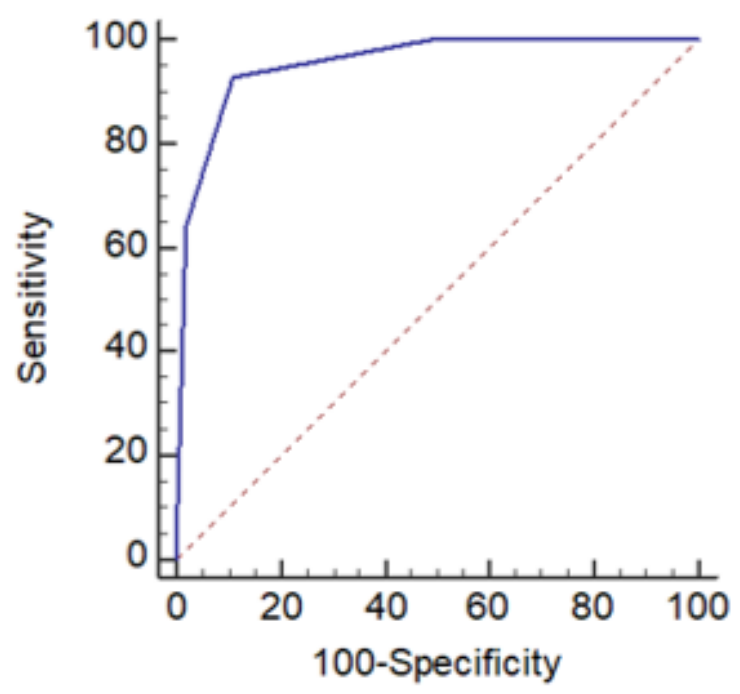

Figure3-2 NCSE poor prognosis

Figure 3

NCSE hospital deaths and poor prognosis 